\title{
Patient Monitoring During Magnetic Resonance Imaging Exams by Means of Ballistocardiography
}

\author{
Karen Meyer zu Hartlage, Enrico Pannicke, Stefano Orsolini, \\ Georg Rose, Ralph Vick, Johannes Krug Passand \\ Forschungscampus STIMULATE, Department of Medical Engineering, \\ Otto-von-Guericke-University Magdeburg, Germany
}

\begin{abstract}
Magnetic resonance imaging (MRI) has become indispensable in modern medicine. In many cases, it is necessary to monitor the vital signs of the patient during an MRI exam, particularly the heart rate. The MRI's environment often makes it difficult to acquire reliable physiological signals, especially the electrocardiogram (ECG). In addition, applying the monitoring hardware requires additional patient preparation time. In order to avoid these drawbacks, this work makes use of the ballistocardiographic (BCG) effect for obtaining a heart rate estimate.

To measure body motions caused by the BCG effect, an inflated air cushion was integrated into the MRI's patient table. An external pressure sensor detected the pressure changes caused by the BCG effect. A synchronously acquired ECG was used as a reference. Five subjects were included in this preliminary study. The measurements were performed inside a $3 \mathrm{~T}$ MRI scanner.

Taking the ECG as reference or gold-standard, heart beats in the BCG were detected with sensitivity of $91.7 \%$ and a positive predictive value of $93.9 \%$.

This preliminary study showed the feasibility of using $B C G$ signals for patient monitoring during MRI exams. In conclusion, this approach enables patient monitoring during MRI exams and eliminates the time-consuming patient preparation.
\end{abstract}

\section{Introduction}

Magnetic Resonance Imaging (MRI) is an excellent method for non-invasively visualizing human organs and tissues. It allows tomographic imaging without using ionizing radiation.

MRI is used for diagnostic purposes as well as for guiding minimally invasive interventions. In many cases, both procedures require a monitoring of the vital signs of the patient, particularly the heart rate and the heart rhythm. These parameters are usually obtained from the patient's electrocardiogram (ECG) or via pulse oximetry. Both techniques, however, require an additional and timeconsuming preparation of the patient.

The electrical cables and components required for the MRI measurements can cause MR imaging artifacts. In addition, the ECG is severely distorted during MR imaging due to the different magnetic fields which are present inside the MRI scanner.

While the interaction between the blood flow and the static magnetic field causes the magnetohydrodynamic (MHD) effect, the switched gradient magnetic fields induce additional distortions inside the human body and the ECG cables [1].

The aim of this work was to develop a completely MRcompatible system for monitoring a patient's heart rate based on the BCG effect. The developed system does not require any wiring of the patient which enhances the comfort of an MRI exam and reduces the time required for patient preparation.

\section{State of the art}

\subsection{Current clinical practice}

In current clinical practice, the heart rate monitoring during an MRI exam is performed by a dedicated ECG system or by pulse oximetry.

The ECG setup includes electrodes, cables and electronics for signal transfer, signal processing and trigger generation. The patient preparation includes skin preparation such as shaving chest hair and cleaning with special gels, as well as electrode positioning [2].

In addition, ECG signal acquisition and analysis is hampered by the different magnetic fields distorting or superimposing the ECG [1]. Hence, a reliable ECG processing for QRS detection and heart rate estimation is not possible in all cases. In these cases, pulse oximetry is the most common replacement. 


\subsection{Heart rate measurement via a pneu- matic method}

The contraction of the heart muscle and the ejection of blood from the ventricles into the circulatory system cause slight body movements. In the context of this work, all these movements caused by the cardiac activity are referred to as ballistocardiographic (BCG) effect. Among others, these movements can be detected by pneumatic pressure variations. Watanabe et al. proposed such a pneumatic method for measuring differnt vital signs [3]. They used an air cushion attached to a pressure sensor and placed them under a bed mattress. Their aim was to determine heart rate and respiration rate, body movement and snoring during sleeping. This method does not require a direct connection or electrical wiring of the patient.

\section{Material and methods}

\subsection{Measurement setup}

This work makes use of the BCG effect for obtaining a heart rate estimate similiar to the approach described by as Watanabe et al. [3]. In order to maintain full MRcompatibility, we adopted and modified this setup.

Figure 1 shows the experimental setup used in the MRI scanner to acquire the physiological signals from different subjects using a pressure cushion integrated in the MRI patient table. For the measurement of the pressure or BCG signal, an inflated air cushion (IPT GmbH, Weimar, Germany) was integrated into the upper area of the MRI patient table. The air cushion was connected to an external pressure sensor (Board Mount Pressure Sensor, Honeywell, Charlotte, North Carolina, USA) outside the MR scanner room using a flexible tube (length: $4 \mathrm{~m}$, diameter: $3 \mathrm{~mm}$ ). All electronics were also placed outside the MR scanner room which made the setup completely MR-compatible. Data acquisition and evaluation were performed by using a single-board computer (Raspberry Pi 3 Model B, Raspberry Pi Foundation, Cambridge, United Kingdom) with a sampling frequency of $1 \mathrm{kHz}$. The sensor was connected to the computer via an $\mathrm{I}^{2} \mathrm{C}$-bus. In order to suppress baseline drifts caused by respiration and high frequency signal components, a digital band-pass filter with the lower and upper cutoff frequencies of $0.8 \mathrm{~Hz}$ and $5 \mathrm{~Hz}$ was applied to the pressure signal using Matlab (The MathWorks, Inc., Natick, Massachusetts, USA). A preliminary peak detection was performed using Matlab's standard peak detection algorithm.

In addition to the BCG signal, the subject's ECG signal was acquired and used as a reference or gold-standard for the evaluation purposes. The ECG was synchronously measured using an in-house developed, battery powered, wireless ECG system (sampling frequency $1 \mathrm{kHz}$ ). Both

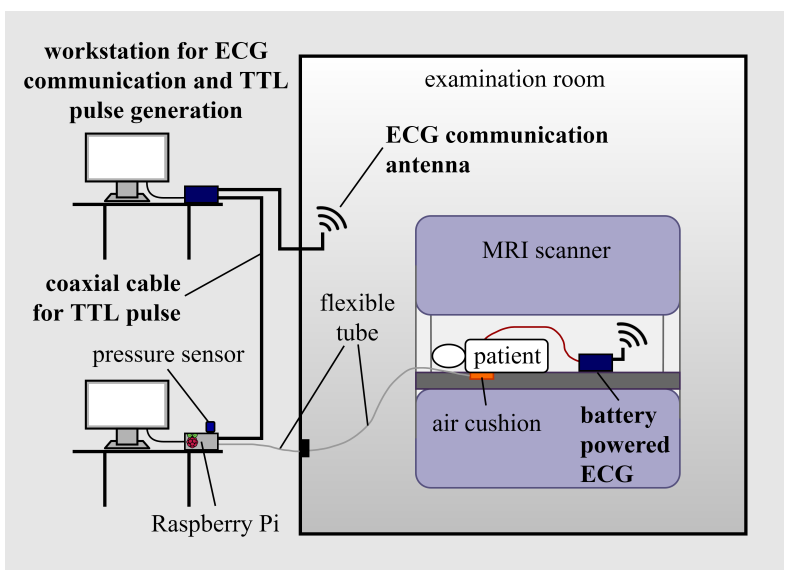

Figure 1: Experimental setup used for the acquisition of the BCG and the reference ECG signals. The BCG signals were acquired by an air cushion integrated into the patient table.

acquisition systems (BCG and ECG) were synchronized by a TTL signal generated by the ECG acquisition module.

\subsection{Experimental verification}

The feasibility of heart rate estimation with the developed setup was verified in a preliminary study including five subjects (two females, three males). The subjects were between 21 and 30 years old, had a weight of $65 \pm 15 \mathrm{~kg}$ and did not have any known cardiac diseases. The measurements were performed inside a $3 \mathrm{~T}$ MRI scanner for one minute. The subjects were asked to remain in resting positions and to breathe normally. The cushion was placed in the position of the upper chest.

The ECG's QRS-complexes were manually annotated by two different ECG experts in order to use the ECG as a reference for evaluating the peaks or beats detected in the BCG signal. For each subject, the sensitivity (Se) and the positive predictive value $(+\mathrm{P})$ were computed for the $\mathrm{BCG}$ signal.

\section{4. $\quad$ Results}

Figure 2a shows an exemplary, unfiltered BCG signal. The high amplitude, low-frequency oscillations (approximately $0.2 \mathrm{~Hz}$ ) were caused by the subject's respiration. The peaks occurring approximately every second were caused by the cardiac motion or activity. Figure $2 \mathrm{~b}$ shows both signals (ECG and BCG) acquired outside the MRI scanner. The BCG's peaks occur in close proximity of the ECG's T-wave. Figure 2c shows the ECG acquired inside the MRI scanner. Due to the MRI's static magnetic field, this ECG is superimposed by the MHD effect. Finally, 


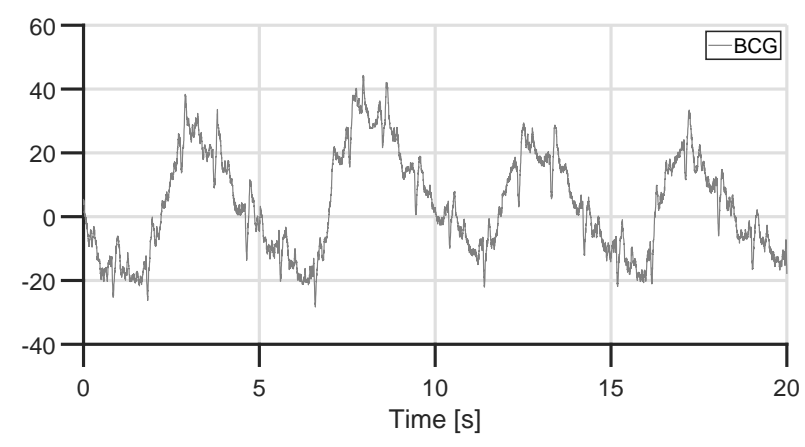

(a) Raw BCG signal.

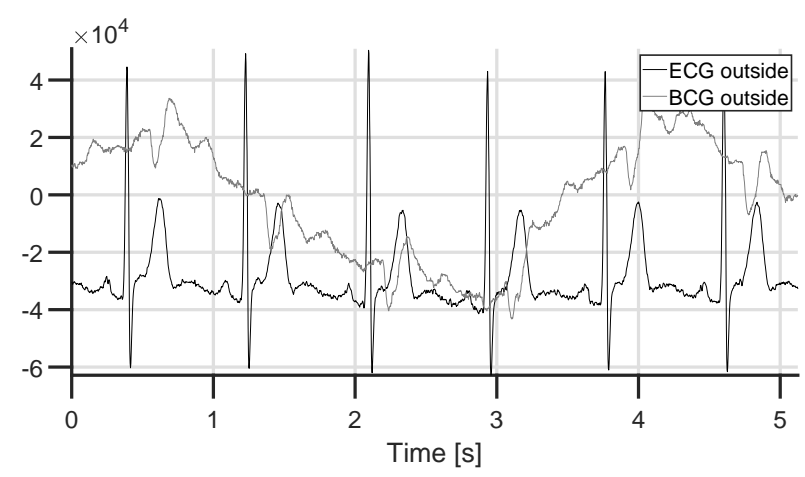

(b) ECG and BCG signals acquired outside the MR scanner.

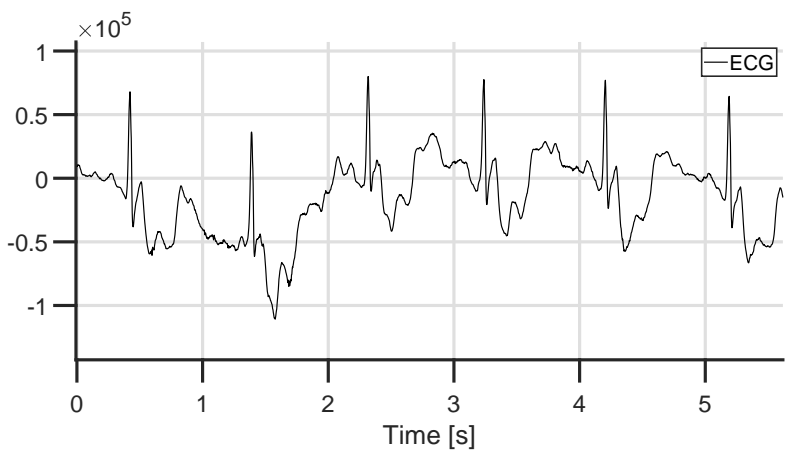

(c) ECG signal acquired inside the MR scanner.

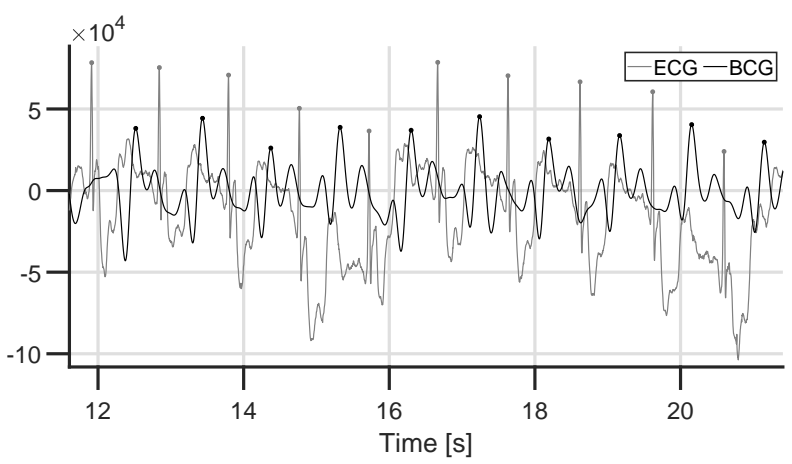

(d) ECG and band-pass filtered BCG signal of subject 3 with detected peaks.

Figure 2: ECG and BCG examples (subject 3).
Fig. 2d show the synchronously acquired ECG and BCG signals as well as the annotated (ECG) and the detected (BCG) beats.

The results of the heart beat detection using the BCG signal are summarized in Table 1.

\section{Discussion and conclusion}

This preliminary study showed the feasibility of using BCG signals for patient monitoring during MRI exams. The main advantage of this setup is the full compatibility with the MRI environment and the reduced patient preparation. Due to the small size of the pressure cushion, it had to be placed quite accurately in order to obtain a sufficient signal quality. Once the subjects were optimally placed on the cushion, only minor artefacts were observed during the BCG measurements.

The quality of the signal and the applied beat detection algorithm were evaluated using a manually annotated ECG signal as a reference. Despite the good BCG signal, several false positive or negative beats were detected in each subject. This was due to the simple peak detection algorithm which was not optimized for the specific BCG signal properties.

In conclusion, the described system eliminates the timeconsuming patient preparation and offers an opportunity to estimate the heart rate by analyzing the pressure signal based on the BCG effect. Furthermore, it is completely MR-compatible and does not have any negative impact on the MR image quality.

In future work, subjects or patients with higher bodymass-index or known cardiac diseases will be included in a further study to investigate the reliability of this method. A dedicated beat detection algorithm for the BCG signal will be developed and implemented in order to improve its reliability and to perform a more reliable beat detection. To improve the causal signal quality, the air cushion will be optimized, either by using one larger or several smaller cushions integrated into the patient table.

Table 1: Results for heart beat detection using the pressure (BCG) signal (Se: Sensitivity, $+\mathrm{P}$ : Positive predictive value).

\begin{tabular}{ccc}
\hline Subject & Se $[\%]$ & $+\mathrm{P}[\%]$ \\
\hline 1 & 86.8 & 90.2 \\
2 & 80.0 & 84.2 \\
3 & 98.4 & 100.0 \\
4 & 94.7 & 96.4 \\
5 & 98.5 & 98.5 \\
\hline Mean & 91.7 & 93.9 \\
\hline
\end{tabular}




\section{Acknowledgement}

The work of this paper was funded by the European Regional Development Fund under the operation number ' $\mathrm{ZS}$ /2016/04/78123' as part of the initiative "Sachsen-Anhalt WISSENSCHAFT Schwerpunkte". The authors have no conflict of interest.

\section{References}

[1] J. Oster and G.D. Clifford, "Acquisition of Electrocardiogram Signals During Magnetic Resonance Imaging," Physiol Meas, vol. 38, no. 7, 2017

[2] T. Niendorf, L. Winter, T. Frauenrath, "Electrocardiogram in an MRI Environment: Clinical Needs, Practical Consid- erations, Safety Implications, Technical Solutions and Future Directions," Advances in Electrocardiograms - Methods and Analysis, January 2012

[3] K. Watanabe, T. Watanabe, H. Watanabe, H. Ando, T. Ishikawa and K. Kobayashi, "Noninvasive Measurement of Heartbeat, Respiration, Snoring and Body Movements of a Subject in Bed via a Pneumatic Method," IEEE Transactions on Biomedical Engineering, vol. 52, no. 12, December 2005

Address for correspondence:

Johannes Passand

OVGU Magdeburg

johannes dot passand at ovgu dot de 